DOI: $10.7242 / 2658-705 \mathrm{X} / 2019.3 .4$

УДК 53.082.2, 532.292, 532.217

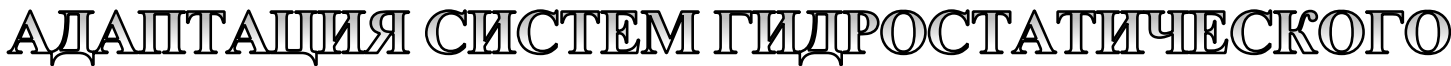

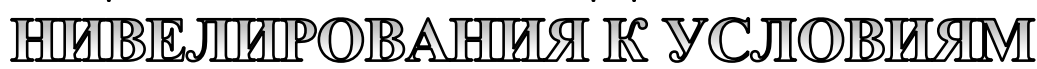

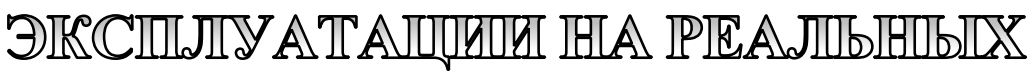

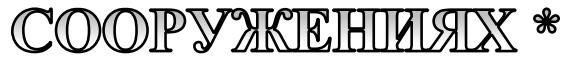

В.В. Епин, Институт механики сплошных сред УрО РАН

С.В. Лекомцев, Институт механики сплошных сред УрО РАН

P.В. Цветков, Институт механики сплошных сред УрО РАН

А.П. Шестаков, Институт механики сплошных сред УрО РАН

Метод гидростатического нивелирования является точным инструментом для определения вертикальных перемещений в составе систем деформационного мониторинга. Условия эксплуатации систем мониторинга накладывают ограничения на использование метода и на достоверность его результатов. В статье рассмотрены случаи из практики, в которых наблюдаются изменения уровня жидкости в гидростатических нивелирах под воздействием окружающей среды. Фундаментальной инженерной проблемой данного исследования является адаптация метода гидростатического нивелирования для использования его в системах деформационного мониторинга реальных зданий и сооружений. Для решения этой проблемы были разработаны модели, описывающие движение жидкости в гидростатических нивелирах, и проведена их экспериментальная проверка. Моделирование позволяет оценивать изменения показаний гидронивелира в зависимости от внешних воздействий (изменение давления воздуха, вибрация, температура). На основании результатов численного решения задачи о тепломассопереносе в системе «шланг - смеситель» дана оценка возможности выравнивания температуры жидкости в гидронивелире путём ее перемешивания. Проведены натурные эксперименты по исследованию принудительного перемешивания жидкости в системе гидростатического нивелирования, которые позволяют снизить температурную погрешность. На основе результатов экспериментальных исследований осуществлен подбор водных растворов для систем гидронивелирования, функционирующих в области отрицательных температур. Проведенные исследования позволяют использовать полученные знания при проектировании систем гидростатического нивелирования, адаптированных для деформационного мониторинга сложных сооружений.

Ключевые слова: деформационный мониторинг, уровень жидкости, гидростатический нивелир, математическое моделирование, эксперимент, тепломассоперенос, теплоотдача, незамерзающая жидкость.

* Работа подготовлена при финансовой поддержке гранта РФФИ №16-48-590025 «Адаптация метода гидростатического нивелирования для систем деформационного мониторинга». 
Организация мониторинга конструкций, зданий и природных объектов невозможна без обеспечения долговременной стабильной работы измерительных систем. Надёжность оценки текущего механического состояния наблюдаемых объектов напрямую зависит от точности проводимых измерений, а достаточное количество накопленных данных позволяет прогнозировать развитие деформационных процессов. Поэтому совершенствование измерительных методик имеет важное значение.

В данной работе проводились экспериментальные и теоретические исследования, направленные на изучение механических и термомеханических процессов, которые происходят в гидростатических нивелирах. Такие приборы применяются в системах деформационного мониторинга различных конструкций $[11,14,16]$, поскольку не требуют прямой видимости между еe элементами. Гидронивелир состоит из наполненных жидкостью сообщающихся сосудов, в которых ее уровень под действием силы тяжести по закону Паскаля устанавливается в одной плоскости. Изменение расстояния по вертикали от этой плоскости до различных точек на объекте исследования определяет поднятие или оседание этих частей. Таким образом, выбирая достаточное количество измерительных сосудов, можно контролировать поле вертикальных перемещений с нужным пространственным разрешением.

В идеальных условиях вертикальные перемещения можно определить с микронной точностью [14], однако при реальных условиях эксплуатации возможны ситуации, когда положение уровня жидкости существенно изменяется. На рисунках приведены случаи из нашей практики, где по различным причинам наблюдается изменения вертикальных перемещений $(\mathrm{U})$, которых на самом деле нет. Эти виртуальные перемещения можно трактовать как погрешность измерительной системы.

В первом примере (рис. 1,a) показано колебание уровня жидкости в одном из датчиков длинного нивелира (общая длина порядка двух сотен метров). На представ- ленном графике видно несколько характерных времен колебаний, наибольшее из которых составляет порядка 8 мин. Чтобы повысить точность в данном случае необходимо или увеличивать время измерения, или оптимизировать геометрические параметры системы. Например, применять измерительные сосуды существенно большего поперечного сечения, чем соединительные трубки. Это уменьшит амплитуду колебаний, но может возрасти время установления уровня жидкости. Во втором примере (рис. 1, б) изменения показаний гидронивелира связаны с изменением давления воздуха между помещениями, где установлены датчики. В течение рабочего дня дверь между помещениями была открыта и наблюдалось одно значение уровня, а ночью дверь плотно закрывалась, поэтому давление воздуха изменялось и, соответственно, уровень жидкости тоже. Чтобы избежать этой проблемы давление воздуха во всех датчиках выравнивается с помощью дополнительной трубки.

На рис. 2, а показано изменение в показаниях прибора вследствие изменения
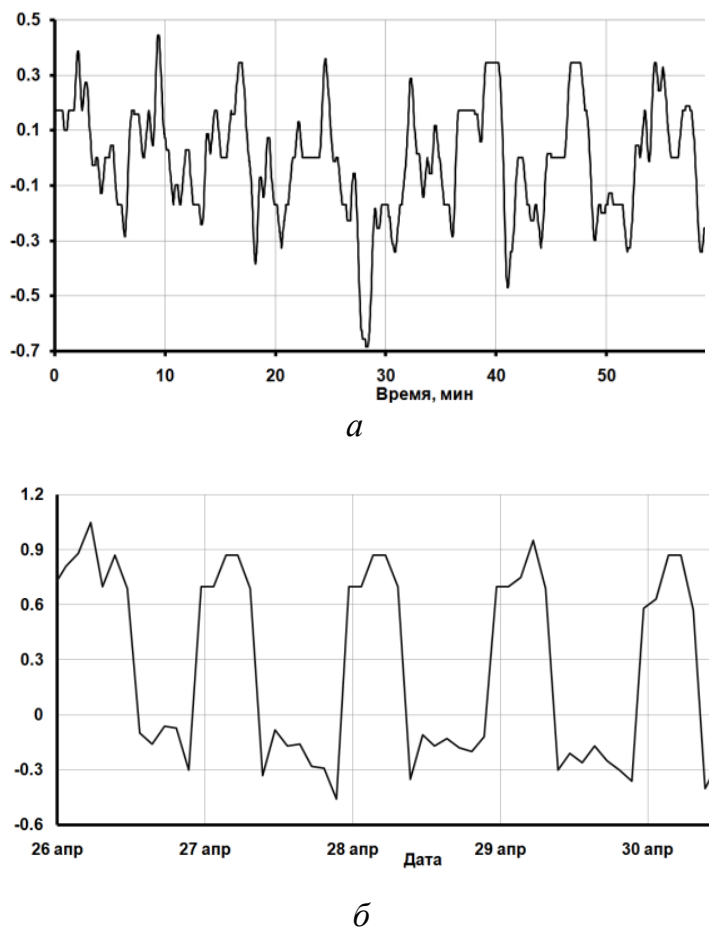

Рис. 1. Примеры погрешности в гидростатических нивелирах: из-за колебаний жидкости (а) и вследствие изменения давления воздуха (б) 
температурного фона в помещении, вызванного полуторанедельным отключением бойлера. В течение 2-3 дней происходит выход параметра на новое значение. Самым эффективным способом борьбы с температурной погрешностью является использование датчиков минимальной высоты и прокладкой соединительной трубки в одном уровне, что вызывает проблемы в некоторых объектах. В четвертом примере (рис. 2, б) можно видеть, что наиболее сильное влияние на показания оказывает образовавшийся в трубке пузырь воздуха. Как известно, жидкость, например вода, содержит растворимый газ, который при повышении температуры оседает в виде маленьких пузырьков на стенках трубки. Этот газ может соединиться в один большой пузырь и полностью перекрыть сечение трубки, таким образом, нарушить гидравлическую связь в нивелире. Поэтому важно не допускать образования пузырей в шлангах гидронивелиров или их устранять. Еще большие проблемы могут возникнуть при замерзании жидкости в отдельных участках гидронивелира.
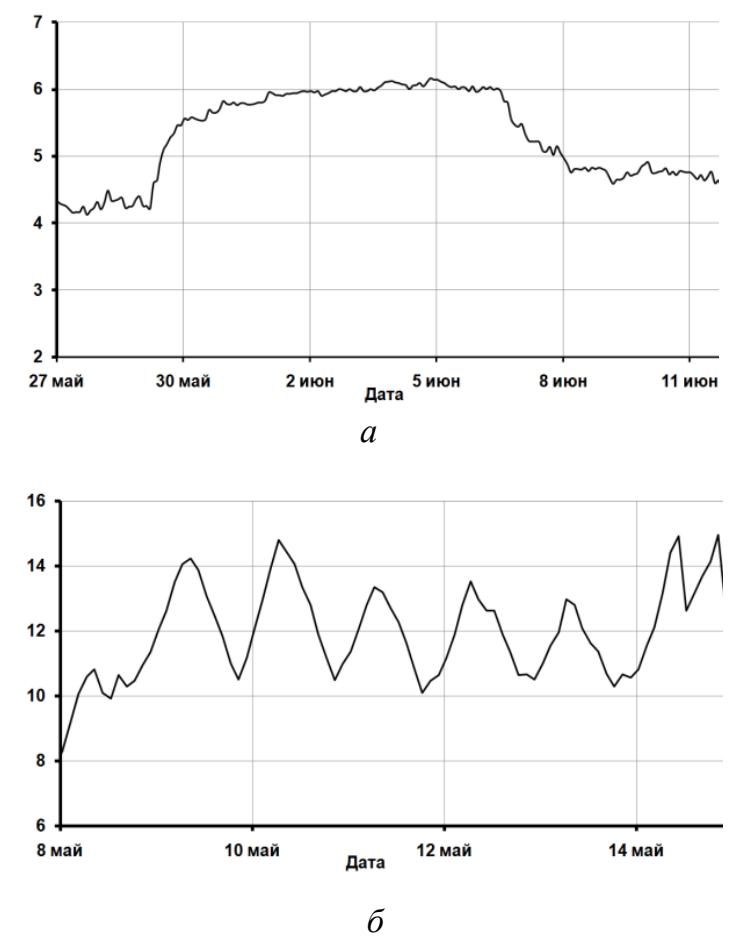

Рис. 2. Примеры погрешности в гидростатических нивелирах: из-за изменения температурного фона (а) и из-за образования воздушного пузыря в трубке (б)
Данные примеры показывают, что уровень жидкости в сосудах не находится строго в одной плоскости, а может изменяться под воздействием различных факторов. Приведенные погрешности измерительной системы могут быть неприемлемы для проводимых исследований, поэтому их необходимо устранять или, по возможности, снижать. Способы снижения погрешностей в принципе известны $[1,3,10]$, однако не все они реализуемы в условиях реальных сооружений. В этой связи была сформулирована цель исследования, которая заключалась в адаптации метода гидростатического нивелирования к условиям эксплуатации в реальных помещениях.

На реальных зданиях и конструкциях, как уже отмечалось, очень сложно обеспечить идеальные условия для измерений соединительные трубки имеют различную длину и располагаются не в одной плоскости, присутствует неустранимый градиент температур (в том числе возможны отрицательные значения), который к тому же изменяется со временем и пр. Для достижения поставленной цели потребовалось решить ряд задач, связанных с описанными выше проблемами: осуществить моделирование процессов движения жидкости в гидронивелирах реальной конфигурации при различных параметрах (давление воздуха, плотность) и проверить адекватность этих моделей с помощью тестовых экспериментов; промоделировать влияние неравномерной температуры на показания гидронивелира; провести численную оценку возможности выравнивания температуры в гидронивелире путем принудительного перемешивания жидкости, а также ее экспериментальную проверку; осуществить выбор жидкостей для работы в области отрицательных температур.

\section{Моделирование поведения жидкости в гидростатическом нивелире}

В литературе $[8,17]$ приводится аналитическое описание переходных процессов в гидронивелирах, как правило, только для 2 датчиков, в том числе и для случая длинных нивелиров [5]. Для боль- 
шего числа датчиков, предлагается производить оценки переходных процессов с помощью аналогии с электрическими цепями: путем замены измерительных и соединительных сосудов на электрическую цепь с элементами R, L и C [12].

B нашей работе поведение жидкости в гидронивелире, состоящем из измерительных сосудов и соединительных сегментов, описывается численно с помощью двух разных моделей: Volume of Fluid [9] и гидравлической модели. Первая модель позволяет оценивать положение поверхности раздела сред жидкости и газа или, другими словами, - уровень жидкости - на основании интегрирования уравнений Навье-Стокса, неразрывности и баланса энергии методом конечных объемов.

Следует отметить, что расчеты по методу Volume of Fluid в среде ANSYS CFX потребовали значительных вычислительных ресурсов, поэтому расчеты производились только для коротких гидронивелиров простых конфигураций. Так, например, была рассмотрена задача о свободных колебаниях жидкости в U-образной трубке, являющейся аналогом 2-датчикового гидронивелира, а также задача о влиянии локального нагрева внутреннего участка $W$-образного нивелира на его показания. Результаты численных расчетов показали, что локальное изменение температуры горизонтальных участков не оказывает влияние на положение свободной поверхности до тех пор, пока не произойдет диффузия нагретой жидкости в вертикальную часть. С другой стороны, изменение температур вертикальных участков оказывает прямое воздействие на показания. При этом не важно, где расположен этот участок: непосредственно под свободной поверхностью или в следующем вертикальном сегменте.

Для реальных конфигураций нивелиров была разработана более простая модель на основе гидравлических соотношений, которая подробно представлена в работах $[6,13]$. Данная модель была протестирована на односегментном $U$-образ- ном нивелире. Результат численного эксперимента показал соответствие с аналитическими формулами для частоты колебаний и декремента затухания [13], однако в эксперименте наблюдались несколько иные значения.

C помощью этой модели также было проведено сравнение свободных колебаний жидкости в нивелире с 4 датчиками при различных его конфигурациях. Полученные результаты [7] показали, что предложенная гидравлическая модель позволяет удовлетворительно оценивать характер переходных процессов и времена затухания колебаний жидкости в многосегментных гидростатических нивелирах и может быть пригодна для проведения оптимизации параметров гидронивелира с целью увеличения его быстродействия [15].

Проведенная по предлагаемой модели оценка влияния неоднородной плотности жидкости (вызванного температурой) в гидронивелире, имеющем многочисленные вертикальные и горизонтальные участки, показала, что изменение плотности жидкости в вертикальных сегментах нивелира отражается на показаниях датчиков [13]. При этом это влияние сказывается независимо от того, насколько далеко расположен вертикальный участок от измерительного модуля. Более того, при различном изменении температуры на разных вертикальных сегментах может наблюдаться как сложение, так вычитание локальных откликов. Этот факт нужно учитывать при проектировании систем гидронивелирования и избегать вертикальных участков в местах с нестабильной температурой.

Результаты моделирования показали, что неустранимый перепад температур в местах прокладки нивелира на объектах со сложной геометрией может вносить существенный вклад в погрешность измерения. Для ее снижения нужно пытаться снизить температурную неоднородность жидкости в нивелире перед проведением измерения, например с помощью принудительной циркуляции. 


\section{Оценка возможности выравнивания температуры жидкости в гидронивелире путем ее перемешивания}

Процедура выравнивания температуры по нивелиру за счет принудительного перемешивания жидкости была рассмотрена на примере тестовой задачи, в которой протяженная трубка (соединительный сегмент нивелира) соединена обоими концами со смесительным баком. Отдельные участки трубки имеют различную температуру. Бак в данной системе служит для ускорения процесса перемешивания. Подробно для 70-метрового нивелира данная задача была рассмотрена в работе [4].

Проведенные расчеты позволили оценить необходимое время перемешивания, позволяющее достичь нужной степени температурной однородности жидкости, а также влияние на него объема смесительного бака. Поскольку измерения гидростатическим нивелиром возможны в отсутствии движения жидкости, также были произведены оценки изменения её температуры после выключения насоса и до полной остановки жидкости. Найденное в численном эксперименте отношение разницы температур в конечный и начальный моменты времени дало оценку степени уменьшения погрешности гидростатического уровня, вызванной неоднородностью внешних воздействий. В примере, рассмотренном в [4], температурную неоднородность удалось снизить на порядок. $\mathrm{C}$ помощью гидравлических формул и зависимости плотности от температуры можно для интересующей геометрии гидронивелира вычислить, к какому снижению погрешности привела данная процедура.

Для фиксации изменения показаний гидронивелира вследствие разницы температуры на вертикальных участках шланга, а также для проверки возможности его устранения путем перемешивания был проведен лабораторный эксперимент, который в какой-то мере воспроизводит ситуацию, возникающую при реальной эксплуатации. Гидронивелир состоял из двух измерительных сосудов, смесительного бака, соединительного шланга общей длиной порядка 30 м, клапанов и насоса, обеспечивающего циркуляцию жидкости в этой системе. Соединительный шланг с внутренним диаметром $d_{i n}=12,5$ мм, толщиной стенки $w=3$ мм, смонтирован таким образом, чтобы иметь два вертикальных участка высотой 1050 мм. Одна вертикальная часть шланга (подъем) расположена в изолированной термокамере, температуру внутри которой можно регулировать. Общая длина шланга в термокамере составила 3 м. Оставшаяся часть нивелира, включая измерительные сосуды, второй вертикальный участок (спуск) и горизонтальные участки находятся в помещении, где температура воздуха остаётся неизменной на протяжении всего эксперимента.

Для снижения взаимного влияния компонентов системы термокамера была расположена в стороне от измерительных сосудов с датчиками. Их объем был выбран намного меньше, чем объем шланга и смесительного бака $(3,7$ л), который применяется для ускорения процессов перемешивания. Это позволяет допустить, что жидкость внутри измерительных сосудов будет обладать такими же свойствами, как и в прилегающей к ней трубке. При проведении процедуры перемешивания жидкость циркулирует по шлангу и переносит тепло, выравнивая таким образом температуру. После окончания процесса перемешивания и перед проведением измерения требуется некоторое время для полной остановки жидкости, за котоpoe её температура может измениться. Измерение температуры жидкости в шланге нивелира проводилось в нескольких точках, три из них располагались в вертикальном участке шланга, находящимся в термокамере.

Эксперимент состоял из 3 этапов: первоначальный нагрев от комнатной температуры, перемешивание жидкости и измерение. В ходе первого этапа жидкость в части нивелира, расположенной в термокамере, нагревалась, что вызывало разли- 
чие показаний датчиков гидроуровня. Во время второго этапа с помощью наcoca осуществлялась циркуляция воды в течение времени, достаточного для выравнивания температуры в шланге. Датчики уровня в это время были перекрыты и не работали. После выключения насоса и открытия клапанов начинался третий этап, во время которого в нивелире прекращались колебательные процессы и производились измерения. Следует отметить, что жидкость начинала вновь нагреваться в тёплой зоне, что вызывало разность показаний гидронивелира.

Проведённый эксперимент продемонстрировал, что температурная неоднородность жидкости при определенной геометрии шлангов гидронивелира действительно является причиной появления значительной погрешности, которая достигла 7,5-8 мм. Её можно компенсировать программно, но необходимо измерять распределение температуры непрерывно по всей высоте вертикальных участков. Наш эксперимент показывает, что даже трех датчиков на небольшом участке может оказаться недостаточно для точного определения градиента температур и его полной программной компенсации. Очевидно, что на реальном объекте такая грубая оценка может привести к неприемлемым результатам.

Предлагаемый метод принудительного перемешивания позволил выровнять температуру и снизить погрешность измерения. Следует отметить, что в течение первых 45 с после начала третьего этапа эта погрешность не превышала 0,25 мм. Именно в это время следует производить измерения вертикальных перемещений с помощью гидронивелира.

Для нивелиров большей протяжённости, располагающихся в неоднородных условиях, необходим комплексный подход, в котором численное моделирование позволит оценить достижимую степень температурной однородности, a значит, и определить погрешность измерения. Принудительное перемешивание перед измерением позволит максимально ее снизить.

\section{Адаптация системы \\ гидронивелирования}

к отрицательным температурам

Важной проблемой для систем гидронивелирования является эксплуатация при отрицательных температурах. В этом случае затруднительно использовать воду наиболее стабильную жидкость, плотность которой меньше всего меняется с температурой в нормальных условиях. По этой причине в качестве незамерзающей жидкости в нивелире можно использовать растворы, содержащие воду и обладающие её преимуществами. Свойства раствора (плотность, вязкость, рабочий диапазон температур) можно изменять, варьируя концентрацию входящих в него компонент. Однако со временем свойства раствора в разных частях нивелира могут изменяться. Например, под воздействием силы тяжести может произойти расслоение (стратификация) жидкости или из-за неравномерного высыхания измениться ее плотность, что может вносить дополнительные искажения в показания прибора. Для обеспечения однородной плотности жидкости потребуются замкнутый в кольцо нивелир, смесительный бак, насос, клапаны и реле. Эта система полностью аналогична описанной выше. Тестовые прогоны жидкостей по ней показали, что с ее помощью также можно успешно выгонять пузыри воздуха, что решает одну из проблем, возникающих при эксплуатации.

Выбор подходящей жидкости, которой реже требуется перемешивание, происходил следующим образом. На неподвижной стене были закреплены три одинаковых U-образных нивелира длиной 4 м, наполненные разными растворами: изопропилового спирта, глицерина и этиленгликоля. Было организовано активное неравномерное высыхание жидкости в нивелире за счет свободного доступа воздуха к одному из измерительных сосудов и перекрытием его к другому. Давление воздуха выравнивалось (то есть второй сосуд также получал доступ к атмосферному воздуху) только на время проведения измерения. За всё время эксперимен- 
та (около 2 месяцев) уровень жидкости в измерительных сосудах, наполненных растворами глицерина и этиленгликоля, понижался со скоростью $0,8 \div 0,9$ мм в сутки. Для раствора изопропилового спирта при таких же условиях наблюдалась более высокая скорость высыхания $1,6 \div 1,8$ мм в сутки. Это привело к необходимости подлива жидкости через 45 дней после начала эксперимента.

За погрешность нивелира, связанную с испарением части жидкости, принято изменение разности показаний в измерительных сосудах со временем. Раствор изопропилового спирта оказался наиме-

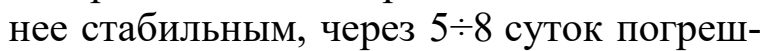
ность уже достигла 0,5 мм, а через месяц эксперимента она превысила 1 мм. У раствора глицерина также наблюдается небольшая тенденция к росту погрешности, через месяц она превысила 0,5 мм, через 50 дней достигла 1 мм. У раствора этиленгликоля первый месяц неравномерного высыхания не наблюдалось явной тенденции к росту погрешности, в течение второго месяца она стала заметна и приблизилась к 0,5 мм. Подробнее с результатами можно ознакомиться в работе [2].

Приведенные результаты свидетельствуют, что раствор этиленгликоля (анти- фриз) лучше других подходит на роль незамерзающей жидкости для гидронивелиpa. Растворы глицерина также не требует еженедельного перемешивания, но они обладают большей вязкостью, что может быть важно при проектировании системы гидростатического нивелирования. Растворы изопропилового спирта быстрее испаряются, поэтому им требуется более частое перемешивание.

\section{Заключение}

Проведенные исследования позволяют повысить точность измерений и расширить границы применения систем гидростатического нивелирования, используемых для деформационного мониторинга сооружений.

В Пермском крае значительная часть граждан проживает на потенциально опасных территориях (карст, техногенные аварии), подверженных осадкам земной поверхности. Вследствие этого здания могут быть подвержены непроектным нагрузкам. Для контроля за состоянием зданий и земной поверхности требуется большое количество относительно простых и надежных систем мониторинга. Её измерительной частью могут быть предлагаемые многодатчиковые гидростатические нивелиры.

\section{Библиографический список}

1. Васютинский И.Ю. Гидронивелирование // - М.: Недра, 1983. - 124 с.

2. Епин В.В., Цветков Р.В. Исследование неравномерного высыхания некоторых водных растворов для систем гидростатического нивелирования// Научно-технический вестник Поволжья. - 2018. - № 9. C. 70-72.

3. Жуков Б.Н. Влияние температурных факторов на точность измерений превышений гидростатическими приборами и системами // Вестник Сибирской государственной геодезической академии. - 1999. - № 4. - С. 42-47.

4. Лекомцев С.В., Цветков Р.В. Оценка возможности выравнивания температуры жидкости в гидронивелире путём перемешивания // Вычислительная механика сплошных сред. - 2018.- Т. 11. № 2.- С. 202-213.

5. Манукин А.Б., Казанцева О.С., Бехтерев С.В., Матюнин В.П., Калинников И.И. Длиннобазисный гидростатический нивелир // Сейсмические приборы. - 2013. - Т. 49. - № 4. - С.26-34.

6. Цветков Р.В., Епин В.В., Лекомиев С.В., Шестаков А.П. Исследование движения жидкости в многосегментном гидронивелире // Научно-технический вестник Поволжья. - 2016. - № 6. - C.92-94.

7. Цветков Р.В., Епин В.В. Экспериментальное исследование переходных процессов в многосегментном гидронивелире // Научно-технический вестник Поволжья. - 2017. - №5. - С. 109-111.

8. D'Oreye $N$., Zurn $W$. Very high resolution long-baseline water-tube tiltmeter to record small signals from Earth free oscillations up to secular tilts // Rev. Sci. Instrum. - 2005. -Vol. $76-024501$.

9. Hirt C.W., Nichols B.D. Volume of fluid (VOF) method for the dynamics of free boundaries // Journal of Computational Physics. - 1981. - Vol. 39 (1). - P. 201-225.

10. Pellissier P.F. Hydrostatic Leveling Systems // IEEE Transactions. - 1965. - Vol. 12. - № 3. - P. 19-20. 
11. Shardakov I.N., Shestakov A.P., Tsvetkov R.V., Yepin V.V. The hydrostatic level method for continuous monitoring of building foundations // Solid State Phenomena. - 2016. - Vol. 243. - P. 105-111.

12. Singatulin S., Volk J., Shiltsev V., Chupyra A., Medvedko A., Kondaurov M. High precision double tubed hydrostatic leveling system for accelerator alignment application // Proceedings of 9th IWAAA06. - USA: Menlo Park, 2006. - FR005.

13. Tsvetkov R.V., Yepin V.V., Shestakov A.P. Numerical estimation of various influence factors on a multipoint hydrostatic leveling system // IOP Conf. Ser. Mater. Sci. Eng. - 2017. - Vol. 208. - 012046.

14. Volk $J$. [et al.] Hydrostatic level sensors as high precision ground motion instrumentation for Tevatron and other energy frontier accelerators // J. Instrum. - 2012. - Vol. 7. - P01004.

15. Yepin V.V., Tsvetkov R.V., Shardakov I.N., Shestakov A.P. Estimation of hydrostatic level parameters for measuring vertical displacement fields of structures on the test stand // AIP Conference Proceedings. 2018. - Vol.2053. -040104.

16. Yin Z.Z. Application of hydrostatic leveling system in metro monitoring for construction deep excavation above shield tunnel // Appl. Mech. Mater. - 2013. - Vols. 333-335. - P. 1509-1513.

17. Zhang C., Fukami K., Matsui S. Primary hydrokinetics study and experiment on the hydrostatic leveling system // Proceedings of the $7^{\text {th }}$ Int. Workshop on Accelerator Alignment. - Japan: Spring-8, 2002. - P. 297-307.

\title{
THE ADAPTATION OF THE HYDROSTATIC LEVELLING SYSTEM TO THE OPERATING CONDITIONS AT REAL STRUCTURES
}

\author{
V.V. Yepin, S.V. Lekomtsev, R.V. Tsvetkov, A.P. Shestakov \\ Institute of Continuous Media Mechanics UB RAS
}

The hydrostatic leveling method as part of deformation monitoring systems is an accurate tool for measuring vertical displacements. However, the operating conditions of the monitoring systems impose restrictions on the use of the method and reliability of its results. The article deals with the cases of practice in which there are errors in liquid level under the influence of the environment. The fundamental engineering problem of this study is the adaptation of the hydrostatic leveling method to the systems for deformation monitoring of real buildings and structures. To solve this problem, a number of models have been developed to describe the movement of liquid at hydrostatic levels, and their experimental verification has been performed. The simulation allowed to evaluate changes in the readings of the hydrostatic level depending on the external action (changes in air pressure, vibration, temperature). The results of the numerical solution to the problem of heat and mass transfer in the «hose-mixer» system have been used to estimate the possibility of equalizing the temperature of the liquid at a hydrostatic level by its stirring. Field experiments were conducted to study the forced liquid stirring in a hydrostatic leveling system, which allow marked reduction of the temperature error. Based on the results of experimental studies, the selection of liquids for hydro-leveling systems operating in the negative temperature range has been carried out. The knowledge obtained from the conducted studies can make up framework for designing hydrostatic leveling systems adapted for the deformation monitoring of complex structures.

Keywords: deformation monitoring, liquid level, hydrostatic level, mathematical modeling, experiment, heat and mass transfer, non-freezing liquid.

\section{Сведения об авторах}

Епин Валерий Валерьевич, младший научный сотрудник лаборатории механики функциональных материалов Институт механики сплошных сред УрО РАН - филиал Пермского федерального исследовательского центра УрО РАН (ИМСС УрО РАН), 614013, ул. Академика Королева, 1; e-mail: epin.v@icmm.ru

Лекомцев Сергей Владимирович, кандидат физико-математических наук, и.о. заведующий лабораторией механики функциональных материалов ИМСС УрО РАН; e-mail: lekomtsev@icmm.ru Цветков Роман Валерьевич, кандидат технических наук, научный сотрудник лаборатории интеллектуального мониторинга ИМСС УрО РАН; e-mail: flower@icmm.ru

Шестаков Алексей Петрович, младший научный сотрудник лаборатории интеллектуального мониторинга ИМСС УpО РАН; e-mail: shap@icmm.ru 